\title{
Measurement of an electroretinogram signal and display waves on graphical user interface by laboratory virtual instrument engineering workbench
}

\author{
Mustafa F. Mahmood, Huda Farooq Jameel, Mayss Alreem Nizar Hammed \\ Department of Medical Instrumentation Techniques Engineering, Electrical Engineering Technical College, \\ Middle Technical University, Baghdad, Iraq
}

\begin{tabular}{l} 
Article Info \\
\hline Article history: \\
Received Sep 25, 202 \\
Revised Dec 5, 202 \\
Accepted Dec 17, \\
\hline Keywords: \\
AD624AD-model \\
Arduino \\
DTL electrode \\
Electroretinogram \\
LabVIEW
\end{tabular}

\begin{abstract}
The electroretinogram (ERG) is an electrophysiological recording method that measures the retinal electrical potential. The electrical reaction is quantified by electrical interaction of the indicator electrode with the cornea or at various levels inside the retina. However, such ERG systems suffer from certain limitations and challenges, such as high cost, low a/b-wave amplitude, and the outcomes do not provide any information about patients. In this work, we designed and implemented a real-time prototype for an ERG system for measuring eye waves via diode-transistor logic (DTL)electrode and AD624AD-model. In addition, a graphical user interface (GUI) via virtual instrument engineering workbench (LabVIEW) was used. The developed system achieved high amplitude for ERG a/b-waves of about 100 and $700 \mathrm{mV}$. In terms of a/b-waves in the system, the findings show that this study has good results for optimizing the measurement of ERG signals. The method showed satisfactory accuracy of about $92.5 \%$ for 10 participants aged 20-60 years and comprising both genders.
\end{abstract}

This is an open access article under the CC BY-SA license.

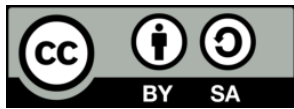

\section{Corresponding Author:}

Mustafa F. Mahmood

Department of Medical Instrumentation Techniques Engineering

Electrical Engineering Technical College, Middle Technical University

Baghdad, Iraq

Email: mustafa.falah@mtu.edu.iq

\section{INTRODUCTION}

Numerous photoreceptor cells are activated when a light flash lands on the retina. The electroretinogram (ERG) records the temporal evolution of this mechanism, which includes changes in interlayer currents that determine variations in ERG. This test is used for diagnosing irregular retina activity, which is the part of the eye that senses light. This examination studies the rods, cones, and light-sensitive cells of the eyes. An electrode is positioned on the cornea head during the test to measure the electrical response of the retinal cells to light. An ERG is useful for determining hereditary and acquired retina disorders. An ERG may also be helpful for deciding whether retinal surgery is preferred to other forms of ocular surgery. For example, cataract surgery may help improve a patient's eyesight. Retinitis pigmentosa, Leber's congenital amaurosis, choroideremia, Usher's syndrome, night blindness, and cone dystrophies are some of the disorders for which the doctor may examine [1]. The retina is positioned on the back of the eye, representing the sensory portion of the eye [2]. The ERG is a prospect for calculating the electrical activity of retinal cells when the eye is stimulated by light of adequate intensity, where the amount of current exterior to the eye can be recorded as a voltage drop on the terminal of the electrode that relates with the eye, then a signal is sent across the cable electrode. The ERG test usually requires a light source, electrodes, and 
electronic circuits. For children, the test uses diverse shapes, for example, cartoon images [3], [4]. This test is highly necessary to determine the types of diseases affecting the retina [5]. Many papers on laboratory virtual instrument engineering workbench (LabVIEW) analysis of ERG waves have been published in the last decade, and it has become a popular subject among scientists. Some researchers examine a variety of different applications and objectives. However, analyzing ERG wave systems relies on electrodes, operational amplifier type, application software, and a/b-wave amplitude.

Umashankar and Panse [6] introduced an ERG system using LabVIEW for measuring a bioelectrical signal of the human retina. The system consisted of a simulation ERG signal, signal measurement, analysis, ERG signal display, and peak values. The experimental results for system showed that anomalies were observed and the simulated ERG was used for examining participants with abnormalities detected. In addition, the a/b-wave amplitude was about $-0.19 \mu \mathrm{V}$ and $+0.5 \mu \mathrm{V}$, respectively. Vijayprasath et al. [7] presented an electrooculography (EOG) system using LabVIEW for measuring waves from eyes. The system involved an electrode, filters, amplifiers, LabVIEW Ni-DAQ, and a laptop or personal computer. The experimental results revealed that the system analyzed data in real-time using a circuit designed in LabVIEW, and the results were displayed on the personal computer (PC). Mamatha et al. [8] studied EOG and electroencephalogram (EEG) signals using several programs such as Multisim and LabVIEW in smart sensor design and analysis of the eye and brain-machine interface. The system comprised a filter, amplifiers, and oscilloscope to display the results. The experimental results revealed that the system had $90 \%$ accuracy for analyzing EOG and EEG signal data. Carpi and Tomei [9] reported on detected eye ERG by using electrodes from several non-invasive methods. The system consisted of electrodes, amplifiers, and oscilloscopes to display the results. The experiment was conducted on pigs, where the device was implanted in the chest. The experimental results revealed that the system recorded the Vpp using electrodes. In addition, the CT electrode performed better than the Henkes corneal electrode for recording amplitudes. Furthermore, the a/b-wave amplitude was about $-100 \mu \mathrm{V}$ and $+220 \mu \mathrm{V}$, respectively.

Esakowitz et al. [10] presented an ERG system using six types of electrodes for measuring the bioelectrical signal of the human eye. The system consisted of electrodes, amplifiers, and recorder waves to display the peak values. The experimental results showed that the Burian-Allen electrode was better than other electrodes in terms of amplitude and efficiency, which were $471 \mu \mathrm{V}$ and $100 \%$, respectively. The a/bwave amplitude was about $-20 \mu \mathrm{V}$ and $+60 \mu \mathrm{V}$, respectively. The system used several types of electrodes. Robles-Camarillo et al. [11] presented an ERG system for measuring the electrical signal of the human eye. The system involved electrodes, an adaptive model, and an electronic system. The experimental results showed that the standard ERG tests improved visual acuity and increased electrical b-wave response when applied to patients with low vision produced by central retinal artery occlusion. The a/b-wave amplitude was about $-160 \mu \mathrm{V}$ and $+400 \mu \mathrm{V}$, respectively. Brigell et al. [12] designed and implemented an ERG test for measuring the a-wave from eyes. The method involved recording ERGs, sequence and timing, scotopic flash spectral characteristics, scotopic stimulus flash strengths, flash duration, photonic background, and response analysis. The experimental results revealed that the system analyzed the retinal data. In addition, disturbances occurring in the retinal receptors were shown. Furthermore, the a-wave amplitude was about $-300 \mu \mathrm{V}$. McCulloch et al. [13] designed and implemented an ERG system for measuring and detecting rods in a cone mutant in humans. The system involved electrodes, an adaptive model, and an electronic system. The authors studied two areas (i.e., light/dark-adaptive) and eye waves. The experimental results showed that a-wave amplitudes were produced by cones and cones and that b-wave amplitudes were generated mainly by retinal activity in bipolar cells. In addition, the b-wave amplitude was $+150 \mu \mathrm{V}$. The system studied the response for light and dark adaptation. However, it was a prototype model.

Grace et al. [14], the objective of their project was to determine the feasibility, sensitivity, and specificity of a non-sedated handheld cone ERG in children's eyes. The system involved electrodes and an adaptive model. The experimental results showed that the ERG tests differed according to the children's ages. Kim et al. [15] studied several waves detected from mouse eyes (i.e. ERG, optical coherence tomography, objective optoretinography), and reported that photoreceptor dysfunction can be detected early. The system involved a light source, electrode, amplifier, and filter. In addition, the wave was processed with several programs (i.e. MATLAB and LabVIEW). The experimental result showed that the waves could detect the initial phases of retinal degeneration. The a/b-wave amplitude was about $-0.3 \mathrm{mV}$ and $+0.3 \mathrm{mV}$, respectively. The system detected a photoreceptor dysfunction and analyzed several waves. Venkatraman et al. [16] studied ERG analysis to detect rods in a cone mutant in zebrafish. The system involved electrodes, an adaptive model, and an electronic system. The authors studied two areas (i.e. light/dark-adaptive). The experimental results showed that dark-adaptive area was low amplitude compared with the light-adaptive area. In addition, the wild-type fish responded to light better than the other types. The b-wave amplitude was $+500 \mu \mathrm{V}$. The system studied responses in animals. However, it was a prototype used for animals only. 
Quite recently, considerable attention has been paid to medical devices to several factors including the high cost, size and weight of the medical device. In additon, amplitude for ERG waves was low in micro volt. The present study involved for resolve these issuses via using two units: hardware and software units. The hardware unit included an electrode, DC power supply, operation amplifier, filter, printer, and an Arduino Mega 2560 microcontroller. The software unit involved the LabVIEW program and graphical user interface (GUI). The main objective was to optimize the measurement of ERG signals. The contribution of this paper is summarized as follows:

- Designed and implemented a lightweight ERG system.

- Improved the measurement of ERG signals relative to previous related works.

- Detected ERG signals via a sterile diode-transistor logic (DTL) electrode. In addition, the ERG signal was amplified via the AD624AD model in the hardware unit.

- Printed information on a patient's eye via the GUI system in LabVIEW in the software unit.

- Compared the results with previous studies in terms of a- and b-waves.

Following is the rest of the paper. The proposed device design is shown in section 2. Section 3 introduces and defines the module experiment configurations. The overall results and discussion of the method are highlighted in section 4. Compare between current and previous studies two parameters in section 5. Finally, a conclusion and recommendation with observations and suggestions for future studies were presented in section 6 .

\section{SYSTEM MODEL}

The ERG is a recording method for determining the electrical potential of the retina. The electrical contact of the indicator electrode can be used for measuring the electrical reaction. Figure 1 depicts the proposed structure, which consists of two units: hardware and software unit. The hardware unit includes the patient's eyes, electrode, DC power supply, operation amplifier, filter, printer, and microcontroller (i.e., Arduino Mega 2560). The software unit involves a laptop, LabVIEW version 2015 (national instruments), and a GUI. The first unit a signal from the eye is detected via the electrodes. Then, it is amplified with an operational amplifier. Next, filters remove the distortion in the signal from the operational amplifier. The second unit, the signal is input to the laptop via the microcontroller. The result is displayed in the LabVIEW GUI and printed on A4 paper.

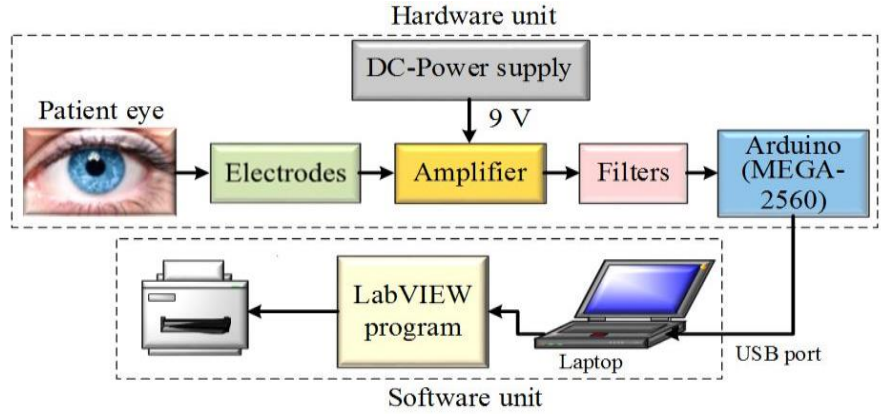

Figure 1. Block diagram for ERG system

\subsection{Hardware unit}

The hardware unit comprises an electrode, DC power supply, operation amplifier, filter, and microcontroller. First, the electrode used involves an active electrode and a ground electrode to ensure the success of the test ERG. In this system, we used a DTL electrode. The active electrode is the main electrode that includes a recording electrode and reference electrode. One characteristic of the reference electrode is that it is an antiseptic electrode that consists of $6.5 \mathrm{~cm}$ silver-impregnated nylon thread affixed to two selfadhesive pads attached to the outer and inner parts of the area around the eye, one terminal that is in contact with the cable connected to the unit. The recording electrode is the electrode in contact with the cornea or the nearby bulbar conjunctiva under the eyelid, which should be used for standard full-field recording, which represents the small affixed positive electrode. The reference electrode should be placed on the skin near the analogous outer part of the area around the eye, representing the large affixed negative posterior electrode. The ground electrode is in contact with the mastoid in the earlobe or forehead locations such as with electrocardiogram (ECG) electrodes. To avoid problems with the signal, it is usually recommended to clean 
the area on which the electrode is placed [17]-[20]. Second, the small amplitude of the eye wave and frequency requires amplification of the signal via an amplifier similar to those used in electrocardiography (i.e. the AD624AD model). The characteristic for this module includes high-accuracy pre-trimmed internal gain resistors. These allow for single connection programming of gains of 1, 100, 200, and 500 [21]. The operation amplifier requires a $9 \mathrm{~V}$ external voltage source, so we used a power supply. Third, the output signal that contains noise and interference was filtered. Here, we used a second-order bandpass filter to filter the signal from the operation amplifier [22]. The microcontroller used in the present study is based on the ATmega2560 processor [23], [24]. It is small, targeted at low power consumption, lightweight, and inexpensive [25], [26]. Also, it is programmed using C+ language with a $16 \mathrm{MHz}$ crystal oscillator [27]. It has 54 digital input/output pins. Note that this number of pins consists of 14 pins used as pulse-width modulation (PWM) outputs, 16 pins used as analog inputs, and four pins used as universal asynchronous receiver transmitter (UART) hardware serial ports [28]-[30].

\subsection{Software unit}

The software unit involves three main components: laptop, LabVIEW [31], [32] and a printer for printing the result. The laptop is connected with the microcontroller via a universal serial bus (USB) cable. The laptop also supplies power to the microcontroller and sends information to LabVIEW via the USB cable. Next, we used LabVIEW for processing and analyzing patient information such as gender and age according to the GUI. This information was printed via a Canon G-3411 wireless printer on A4 paper. Table 1, shows the current experimental technique as well as the test parameters used.

Table 1. The current experiment, the experimental technique and test parameters were used

\begin{tabular}{ll}
\hline \multicolumn{1}{c}{ Parameters } & \multicolumn{1}{c}{ Description } \\
\hline DTL electrode & $6.5 \mathrm{~cm}$ silver-impregnated nylon thread affixed to two self-adhesive pads \\
Amplification- AD624AD model & High-accuracy pre-trimmed internal gain resistors, gains of 1, 100, 200, and 500 \\
Filter & Second-order bandpass filter rang between 0.1 and 3000 Hz \\
Processing & Microcontroller Arduino Mega 2560 \\
Software & - Arduino IDE software \\
& - LabVIEW \\
Devices & - Power supply 9 V \\
& - Storage oscilloscope (model UTD2025CL) \\
& - Canon G-3411 wireless printer \\
Display signal & - Storage oscilloscope (model UTD2025CL) \\
& - GUI in LabVIEW \\
& - Paper A4 \\
\hline
\end{tabular}

\section{EXPERIMENT CONFIGURATION}

The ERG is a recording method that determines the retinal electrical potential. The electrical contact of the indicator electrode can be used to measure the electrical response. The electrode (i.e., reference, record, ground) is placed close to the eye to detect an ERG signal. This detected wave is low-amplitude, therefore requiring amplification via operation amplifiers (i.e., the AD624AD model). The signal from the electrode cable to the AD624AD model to input pin 1 and pin 2 in the same model. Operating this model requires a $9 \mathrm{~V}$ external voltage source via a DC power supply. In the model, pin 3, 11, and 13, and pin 12 and 16 are attached to obtain high amplification. In addition, the output signal from the connection is sorted in pins 9 and 10. Subsequently, the signal that emerges from the operations amplifier contains information and noise. To remove this noise, we used a filter circuit to filter the signal via the passive second-order bandpass filter between 0.1 and $3000 \mathrm{~Hz}$. Finally, the final wave is inserted into the microcontroller to link it with the laptop through the analog pin 8 in the microcontroller. In addition, the system is interfaced with a microcontroller (i.e., Arduino Mega 2560) via LabVIEW 2015 and Arduino 1.8.13. The Arduino version was set according to the interface with LabVIEW, and we also setup other libraries: LabVIEW Interface for Arduino, LIFA_Base, and VI Package Manager.

The patient is connected to the ERG system via the DTL electrode and ground electrode, which detect the ERG signal. Show as in Figure 2(a), the DTL-electrode and ground electrode attached near the patient's eye in terms of active, record and ground electrode. Then, the received signal is entered into the ERG system to perform signal amplification and filtering operations Figure 2(b). The ERG signal can be displayed in two ways: an oscilloscope and via LabVIEW. The system is interfaced with a laptop via the microcontroller to process and display a wave in the GUI result Figure 2(c). The circuit components for the ERG system are illustrated in Figure 2(d). Each study site's protocol was authorized by an independent review board or ethics committee prior to the start of the study. All of the patients signed an informed consent form. 
LabVIEW involves two windows: front plant and block diagram. The front plant design includes the name of the test, patient's name and age, date of the test, screen to show a wave, input port for the microcontroller, and print switch Figure 3(a). The block diagram design involves input port properties (i.e., visa, port rate, microcontroller type, port type), the Arduino program (i.e., initial and type of signal according to [analog or digital signal]). In addition, the date of the test and the patient's name and sex can be printed out on paper. The data rate of 115,200 bps was used to improve the transmission time between the hardware and software units. We used an analog input pin to attach with A8 at the microcontroller. Furthermore, we used it while the loop was used to continue reading from the same input pin Figure 3(b). Finally, we built an independent application that does not work on LabVIEW, and installed it on a PC on which LabVIEW was not installed. The test was performed on 10 people aged 20-60 years. Figure 3(c) shows the results based on each AD624AD model and DTL electrode. In order to record rod b-wave and cone a-wave responses, patients groups were exposed to (25-30) minutes and (8-10) minutes for dark and light adaptation, respactivly. The photoreceptors and this response represent the ERG signal. Then, we detected this response through the DTL electrode and performed the amplification and filtering operations in this system. This was followed by sending the signal to the microcontroller to transfer it into the laptop and display it on LabVIEW through the GUI.

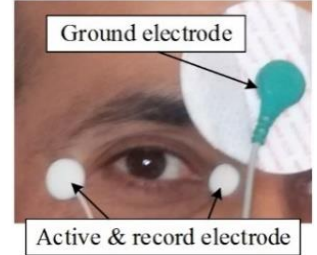

(a)

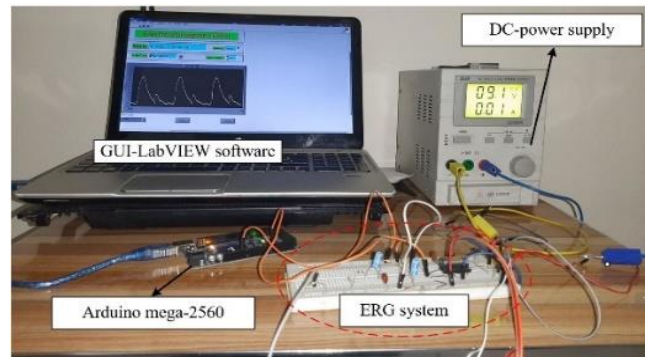

(c)

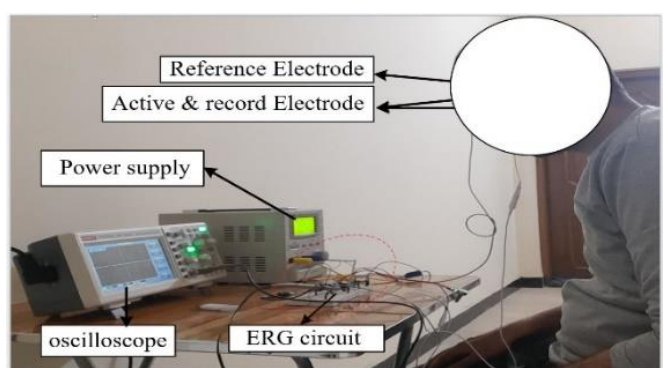

(b)

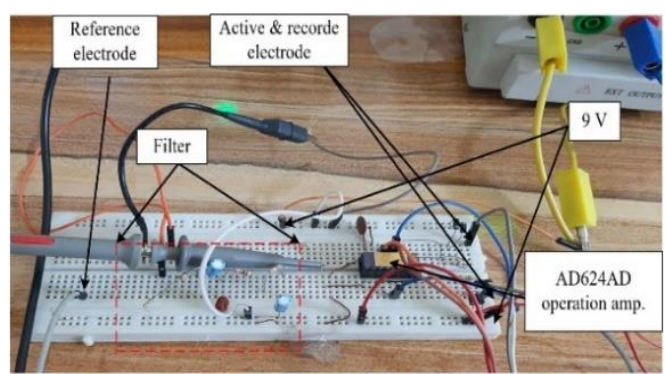

(d)

Figure 2. Snapshot the ERG based: (a) ERG electrodes, (b) system connection for the patient, (c) system interface with software unit, (d) the electronic circuit design for the system

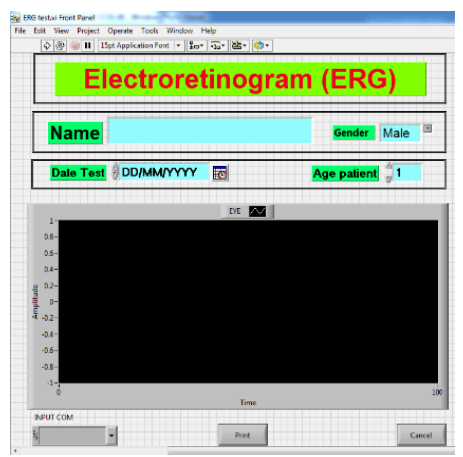

(a)

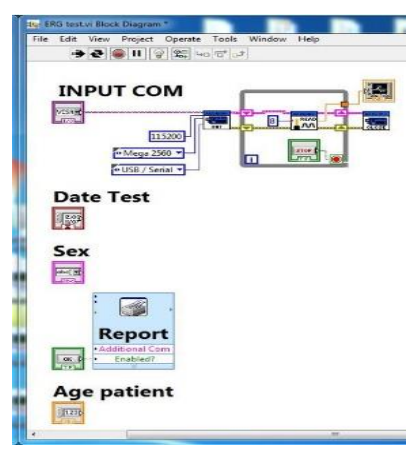

(b)

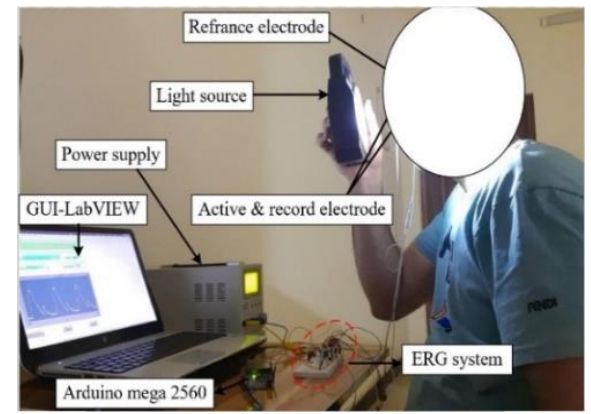

(c)

Figure 3. The design of LabVIEW windows: (a) front plant, (b) block diagram, and (c) snapshot an ERG test to display a result in GUI LabVIEW software 


\section{RESULTS AND DISCUSSION}

In this section, the experimental results of the proposed system. The signal was shown using two methods, the first using the oscilloscope and the second using the LabVIEW program. It is presented as follows.

\subsection{ERG wave via oscilloscope}

This section shows the ERG wave via a storage oscilloscope once the experimental control has been achieved (model UTD2025CL). In Figure 4, the $x$-axis shows the times, whereas the $y$-axis shows the amplitude. The system measured the amplitude for ERG waves such as the $\mathrm{a} / \mathrm{b}$-wave based on connected the system in Figure 2(b). The observed amplitude for the a/b-wave was about $100 \mathrm{mV}$ and $700 \mathrm{mV}$, respectively, via the operation amplifier (i.e., model AD624AD) and DTL electrode. The system was measured using an oscilloscope to compare the apparent results with the results that would appear using LabVIEW.

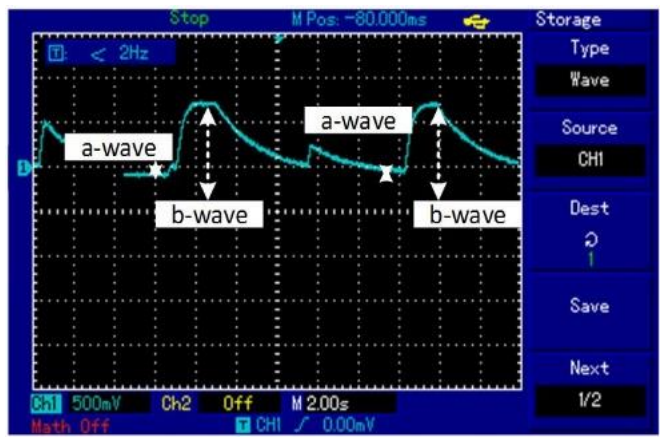

Figure 4. The result for ERG waves-based-oscilloscope

\subsection{ERG wave via LabVIEW}

This section shows the ERG wave via LabVIEW once the experimental control has been achieved show in Figure 5 based on connected the system in Figure 2(c). When the program execution button was run continuously, the signal would appear repeatedly until the stop button was paused, and the signal would stop as the snapshot Figure 5. The ERG signal input to the microcontroller from analog pin A8 via hardware unit also displayed a result in the GUI via LabVIEW in Figure 5. In addition, in LabVIEW showed a result with the patients' details on the GUI, and could print a result on A4 paper or save the result to a PDF file. In LabVIEW, a separate program or application can be created that does not depend on the LabVIEW program, meaning it is possible to install this design as a standalone program on a PC.

The accuracy was evaluated in elderly and young participants, i.e., the 10 people mentioned earlier. The participants tested the system, and each order was repeated five times to review the results and accuracy. The average accuracy was $92.5 \%$. Table 2 presents the results on accuracy for participants $1-10$, with the average of all participants. Each study site's protocol was authorized by an independent review board or ethics committee prior to the start of the study. All of the patients signed an informed consent form.

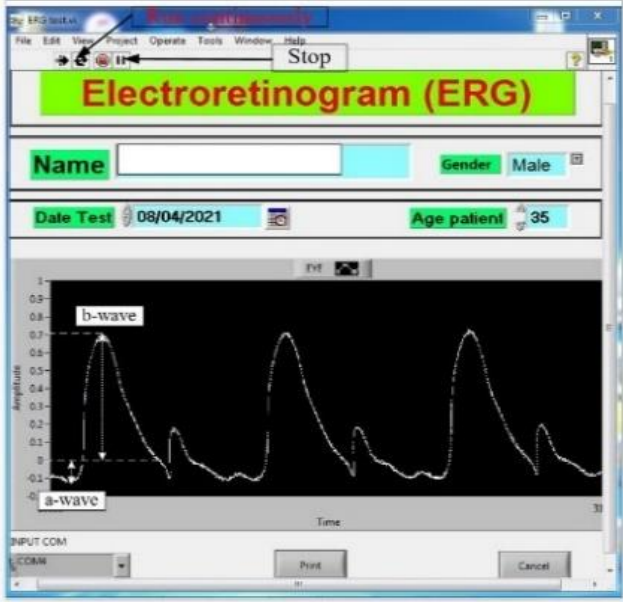

Figure 5. The ERG signal in front plant by LabVIEW 
Table 2. Performance accuracy for ten patients

\begin{tabular}{cclc}
\hline Subject & Age (year) & Gender & Accuracy (\%) \\
\hline 1 & 20 & Male & 99 \\
2 & 22 & Female & 98 \\
3 & 27 & Male & 97 \\
4 & 29 & Female & 94 \\
5 & 33 & Female & 90 \\
6 & 35 & Male & 95 \\
7 & 39 & Female & 99 \\
8 & 44 & Male & 88 \\
9 & 52 & Female & 85 \\
10 & 58 & Male & 80 \\
\multicolumn{4}{l}{ The average accuracy (\%) } \\
\hline \multicolumn{4}{c}{}
\end{tabular}

\section{COMPARISON WITH PREVIOUS WORK}

In this section, we compare our performance metrics with that of previous studies in terms of awaves and b-waves. Table 3 depicts a comparison of the present work and previous studies for $a / b$-wave amplitude. In the present study, the a-wave was $100 \mathrm{mV}$, whereas it was $0.3 \mathrm{mV}$ in [12], [15]. Further, the awave amplitude was $0.16,0.1,0.04,0.02$, and $0.00019 \mathrm{mV}$ in [6], [9]-[11] and [13] respectively. In addition, depicts the comparison between the present work and previous studies for b-wave amplitude. In the present study, the b-wave was $700 \mathrm{mV}$, whereas it was $0.5,0.4,0.3,0.22,0.15,0.06$, and $0.0005 \mathrm{mV}$ in [6], [9]-[11], [13], [15], and [16], respectively. Here, we have summarized several related studies based on the application of the objective, application, implementation environment, and a/b-wave amplitude, as shown in Table 3.

Table 3. Performance metrics of previous works

\begin{tabular}{|c|c|c|c|c|c|}
\hline Ref. & Objective & Application & $\begin{array}{c}\text { Implementation } \\
\text { environment }\end{array}$ & $\begin{array}{l}\text { A-wave amp } \\
\text { in }(-\mathrm{mV})\end{array}$ & $\begin{array}{l}\text { B-wave amp } \\
\text { in }(+\mathrm{mV})\end{array}$ \\
\hline [6] & Objective an analysis of bioelectrical signal & ERG & Simulation & 0.00019 & 0.0005 \\
\hline [7] & Optimize an analysis waves from eyes & EOG & Simulation & N/A & N/A \\
\hline [8] & analysis of eye and brain machine interface & EOG \& EEG & Experimental & N/A & N/A \\
\hline [9] & detected eye electrical wave & ERG & Experimental & 0.1 & 0.22 \\
\hline$[10]$ & Compare electrodes & ERG & Experimental & 0.02 & 0.06 \\
\hline [11] & Optimize analysis of signal an Objective & ERG & Simulation & 0.16 & 0.4 \\
\hline [12] & Objective an analysis waves from eyes & ERG & Simulation & 0.3 & N/A \\
\hline [13] & Objective an analysis waves from eyes & ERG & Simulation & 0.04 & 0.15 \\
\hline$[14]$ & Optimize an analysis an electrical waves for eye & ERG & Experimental & N/A & N/A \\
\hline [15] & Objective an analysis waves from eyes in animal & ERG & Experimental & 0.3 & 0.3 \\
\hline$[16]$ & Objective an analysis waves from eyes in animal & ERG & Simulation & N/A & 0.5 \\
\hline $\begin{array}{l}\text { This } \\
\text { work }\end{array}$ & $\begin{array}{l}\text { Optimize analysis ERG waves via operation } \\
\text { amplifier. }\end{array}$ & ERG & Experimental & 100 & 700 \\
\hline
\end{tabular}

\section{CONCLUSIONS}

This paper introduces the design and implementation of an ERG test for analyzing waves for the eyes to solve high cost, size and weight of the ERG device. Which include hardware and software units. An electrode, DC power supply, amplifier, filter, and microcontroller (i.e., the Arduino Mega 2560) for hardware unit. A laptop, LabVIEW, and a printer for printing the results for software unit. In the experimental results, the DTL electrode and activity amplifier (i.e., the AD624AD model) achieved high amplitude for ERG a/bwaves of about 100 and $700 \mathrm{mV}$, respectively. In addition, the proposed system in a/b waves were researched and compared to those in prior papers to validate wave amplitudes, and it was discovered that these two parameters outperformed previous values. The average accuracy rate was $92.5 \%$, a test was examined in participants aged 20-60 years old and who comprised both men and women. The system was tested by 10 participants, and each order was repeated five times to ensure that the results and accuracy requirements were met. Future work will focus on using wireless technology to transmit and receive data (i.e., Bluetooth, nRF24L01). In addition, we intend to use energy harvesting techniques to operate a device without a power supply via magnetic resonator coupling and capacitor coupling. Furthermore, we will use image processing to obtain the window for the ERG signal and use other types of electrodes.

\section{ACKNOWLEDGEMENTS}

The authors would like to express their gratitude to the Electrical Engineering Technical College at Middle Technical University in Baghdad, Iraq, for assisting them in carrying out the experiments. 


\section{REFERENCES}

[1] M. Ross et al., "Consecutive unilateral recording of the two eyes affects dark-adapted ERG responses, when compared to simultaneous bilateral recording," Documenta Ophthalmologica, vol. 137, no. 3, pp. 183-192, 2018, doi: 10.1007/s10633-0189661-y.

[2] N. R. Galloway, W. M. K. Amoaku, P. H. Galloway, and A. C. Browning, Common Eye Diseases and their Management, London, U.K.: Springer, 2016, doi: 10.1007/978-3-319-32869-0.

[3] D. C. Hood, J. G. Odel, C. S. Chen, and B. J. Winn, "The multifocal electroretinogram," Journal of Neuro-Ophthalmology, vol. 23, no. 3, pp. 225-235, 2003, doi: 10.1097/00041327-200309000-00008.

[4] M. F. K. Değirmenci, S. Demirel, F. Batığlu, and E. Özmert, "Role of a mydriasis-free, full-field flicker ERG device in the detection of diabetic retinopathy," Documenta Ophthalmologica, vol. 137, no. 3, pp. 131-141, 2018, doi: 10.1007/s10633-0189656-8.

[5] J. G. Webster, Medical Instrumentation: Application and Design, New York, USA: John Wiley \& Sons, 2009

[6] Umashankar and M. S. Panse, "Analysis of bioelectrical signal of the human retina (ERG) using LabVIEW," 2010 IEEE Students Technology Symposium (TechSym), 2010, pp. 54-58, doi: 10.1109/TECHSYM.2010.5469191.

[7] S. Vijayprasath, R. Sukanesh, and S. Palanivel Rajan, "Experimental explorations on EOG signal processing for realtime applications in labview," 2012 IEEE International Conference on Advanced Communication Control and Computing Technologies (ICACCCT), 2012, pp. 67-70, doi: 10.1109/ICACCCT.2012.6320743.

[8] M. N. Mamatha, S. Ramachandran, and M. Chandrasekaran, "Smart sensor design and analysis of brain machine interface using labview," 2011 IEEE 3rd International Conference on Communication Software and Networks, 2011, pp. 464-468, doi: 10.1109/ICCSN.2011.6014936.

[9] F. Carpi and F. Tomei, "Non-invasive electroretinography," Biomedicine \& Pharmacotherapy, vol. 60, no. 8, pp. 375-379, 2006, doi: 10.1016/j.biopha.2006.07.002

[10] L. Esakowitz, A. Kriss, and F. Shawkat, “A comparison of flash electroretinograms recorded from Burian Allen, JET ,C-glide, gold foil, DTL and skin electrodes," Eye, vol. 7, pp. 169-171, 1993, doi: 10.1038/eye.1993.36.

[11] D. Robles-Camarillo, L. Nino-de-Rivera, H. Quiroz-Mercado, and M. J. Lopez-Miranda, "Portable transcorneal electrical stimulator system, applied on electrotherapy for low vision patients," 2009 52nd IEEE International Midwest Symposium on Circuits and Systems, 2009, pp. 196-199, doi: 10.1109/MWSCAS.2009.5236118.

[12] M. Brigell, B. G. Jeffrey, O. A. Mahroo, and R. Tzekov, "ISCEV extended protocol for derivation and analysis of the strong flash rod-isolated ERG a-wave," Documenta Ophthalmologica, vol. 140, pp. 5-12, 2020, doi: 10.1007/s10633-019-09740-4.

[13] D. L. McCulloch et al., "ISCEV extended protocol for the stimulus-response series for light-adapted full-field ERG," Documenta Ophthalmologica, vol. 138, pp. 205-215, 2019, doi: 10.1007/s10633-019-09685-8.

[14] S. F. Grace, B. L. Lam, W. J. Feuer, C. J. Osigian, K. M. Cavuoto, and H. Capo, "Nonsedated handheld electroretinogram as a screening test of retinal dysfunction in pediatric patients with nystagmus," Journal of American Association for Pediatric Ophthalmology and Strabismus, vol. 21, no. 5, pp. 384-388, 2017, doi: 10.1016/j.jaapos.2017.06.022.

[15] T.-H. Kim, B. Wang, Y. Lu, T. Son, and X. Yao, "Intrinsic signal optoretinography of rod photoreceptor dysfunction due to retinal degeneration," in Proceedings Ophthalmic Technologies XXXI, vol. 116323, 2021, doi: 10.1117/12.2576897.

[16] P. Venkatraman et al., "Rods Contribute to Visual Behavior in Larval Zebrafish," Investigative Ophthalmology \& Visual Science, vol. 61, no. 12, 2020, doi: 10.1167/iovs.61.12.11.

[17] G. E. Holder, M. G. Brigell, M. Hawlina, T. Meigen, Vaegan, and M. Bach, "ISCEV standard for clinical pattern electroretinography-2007 update," Documenta ophthalmologica, vol. 114, no. 3, pp. 111-116, 2007, doi: 10.1007/s10633-0079053-1.

[18] A. E. Mentzer, D. M .Eifler, F. Montiani-Ferreira, N. Tuntivanich, J. Q. Forcier, and S. M. Petersen-Jones, "Influence of recording electrode type and reference electrode position on the canine electroretinogram," Documenta ophthalmologica, vol. 111, no. 2, pp. 95-106, 2005, doi: 10.1007/s10633-005-4517-7.

[19] T. E. Zhou et al., "Choroidal involution is associated with a progressive degeneration of the outer retinal function in a model of retinopathy of prematurity: early role for IL-1 $\beta$," The American Journal of Pathology, vol. 186, no. 12, pp. 3100-3116, 2016, doi: 10.1016/j.ajpath.2016.08.004.

[20] L. Zhu et al., "All-sealed paper-based electrochemiluminescence platform for on-site determination of lead ions," Biosensors and Bioelectronics, vol. 192, 2021, doi: 10.1016/j.bios.2021.113524.

[21] Analog Devices, Precision Instrumentation Amplifier, 2007. Accessed: Apr. 1, 2021. [Online]. Available: https://www.analog.com/media/en/technical-documentation/data-sheets/AD624.pdf/

[22] G. Liang and F. Chen, "A Compact Dual-Wideband Bandpass Filter Based on Open-/Short-Circuited Stubs," in IEEE Access, vol. 8, pp. 20488-20492, 2020, doi: 10.1109/ACCESS.2020.2968518.

[23] L. Louis, "Working Principle of Arduino and Using It as A Tool for Study and Research," International Journal of Control, Automation, Communication and Systems (IJCACS), vol ,1 ,no. 2.pp. 21-29, 2016, doi: 10.5121/ijcacs.2016.1203.

[24] M. F. Mahmood, S. L. Mohammed, and S. K. Gharghan, "Free Battery-based Energy Harvesting Techniques for Medical Devices," in IOP Conference Series: Materials Science and Engineering, 2020, doi: 10.1088/1757-899X/745/1/012094.

[25] A. D. Deshmukh and U. B. Shinde, "A low cost environment monitoring system using raspberry Pi and arduino with Zigbee," 2016 International Conference on Inventive Computation Technologies, 2016, pp. 1-6, doi: 10.1109/INVENTIVE.2016.7830096.

[26] M. F. Mahmood, S. L. Mohammed, S. K. Gharghan, A. Al-Naji, and J. Chahl, "Hybrid Coils-Based Wireless Power Transfer for Intelligent Sensors," Sensors, vol. 20, no. 9, 2020, doi: 10.3390/s20092549.

[27] M. F. Mahmood, S. L. Mohammed, and S. K. Gharghan, "Energy harvesting-based vibration sensor for medical electromyography device,” Int. J. Electr. Electron. Eng. Telecommun, vol. 9, no. 5, 2020, doi: 10.18178/ijeetc.9.5.364-372.

[28] A. Nayyar and V. Puri, "A review of Arduino board's, Lilypad's \& Arduino shields," 2016 3rd International Conference on Computing for Sustainable Global Development (INDIACom), 2016, pp. 1485-1492.

[29] S. F. Tariq and R. N. Shihab, "Serum and Salivary Immunoglobulins levels in Tobacco Smokers of Baghdad Governorate," Medico Legal Update, vol. 20, no. 3, pp. 1088-1093, 2020, doi: 10.37506/mlu.v20i3.1547.

[30] H. F. Jameel, S. L. Mohammed, and S. K. Gharghan, "Electroencephalograph-Based Wheelchair Controlling System for the People with Motor Disability Using Advanced BrainWear," 2019 12th International Conference on Developments in eSystems Engineering (DeSE), 2019, pp. 843-848, doi: 10.1109/DeSE.2019.00156.

[31] LabVIEW-application. Accessed: May 16, 2021. [Online]. Available: https://www.ni.com/en-lb/shop/labview.html

[32] L. Dello Sterpaio, A. Marino, P. Nannipieri, G. Dinelli, D. Davalle, and L. Fanucci, "A complete EGSE solution for the SpaceWire and SpaceFibre protocol based on the PXI industry standard," Sensors, vol. 19, no. 22, 2019, doi: 10.3390/s19225013. 


\section{BIOGRAPHIES OF AUTHORS}
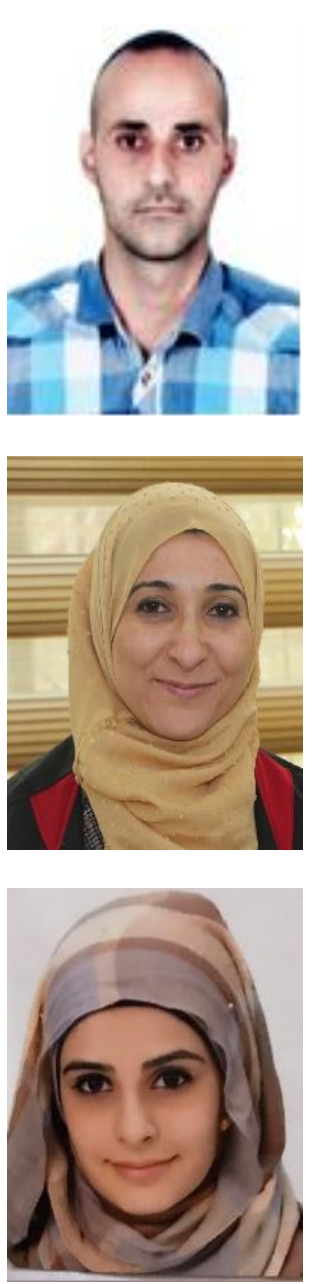

Mustafa Falah Mahmood (D) 8 SC P received his B.Sc. in Medical Instrumentation Techniques Eng. From Middle Technical University, Iraq in 2010. He is with the Department of Medical Instrumentation Engineering Techniques, Electrical Engineering Technical College, Middle Technical University, Baghdad-Iraq, as an Assistant teacher. Has MSc degree in Medical Instrumentation Engineering Techniques, from Electrical Engineering Technical College, Baghdad-Iraq in 2020. Interested in studies that are concerned with wireless energy transmission, alternative energy generation without the use of batteries, the design of medical devices with low cost and high efficiency, biomedical sensors, and microcontroller applications. He can be contacted at email: mustafa.falah@mtu.edu.iq.

Huda Farooq Jameel (D) SD SC P received her B.Sc. in Medical Instrumentation Techniques Eng. from Middle Technical University, Iraq in 2006. She is with the Department of Medical Instrumentation Engineering Techniques, Electrical Engineering Technical College, Middle Technical University, Baghdad-Iraq, as Technical Engineer. In 2020 she has a mater degree Tech. in Medical Instrumentation Engineering Techniques, from Electrical Engineering Technical College, Baghdad-Iraq. She interested in controlling medical device, image processing, and signal processing. She can be contacted at email: huda_baban@mtu.edu.iq.

Mayss Alreem Nizar Hammed (iD 8d SC P received her B.Sc. in Medical Instrumentation Techniques Eng. from Middle Technical University, Iraq in 2013. She is with the Department of Medical Instrumentation Engineering Techniques, Electrical Engineering Technical College, Middle Technical University, Baghdad-Iraq, as Technical Engineer. Has MSc degree in Medical Instrumentation Engineering Techniques, from Electrical Engineering Technical College, Baghdad-Iraq in 2019. Her research is design UWB antenna used for capsule endoscopy. She can be contacted at email: Mayssalreem92@mtu.edu.iq. 